Preprint

UCRL-JC-144249

\title{
Teller Medal Lecture IFSA2001: Problems and Solutions in the Design and Analysis of Early Laser Driven High Energy Density and ICF Target Physics Experiments
}

\author{
M. D. Rosen
}

Lawrence Livermore National Laboratory
This article was submitted to $2^{\text {nd }}$ International Conference on Inertial Fusion Sciences and Applications

Kyoto, Japan

September 9-14, 2001

August 20, 2001 


\section{DISCLAIMER}

This document was prepared as an account of work sponsored by an agency of the United States Government. Neither the United States Government nor the University of California nor any of their employees, makes any warranty, express or implied, or assumes any legal liability or responsibility for the accuracy, completeness, or usefulness of any information, apparatus, product, or process disclosed, or represents that its use would not infringe privately owned rights. Reference herein to any specific commercial product, process, or service by trade name, trademark, manufacturer, or otherwise, does not necessarily constitute or imply its endorsement, recommendation, or favoring by the United States Government or the University of California. The views and opinions of authors expressed herein do not necessarily state or reflect those of the United States Government or the University of California, and shall not be used for advertising or product endorsement purposes.

This is a preprint of a paper intended for publication in a journal or proceedings. Since changes may be made before publication, this preprint is made available with the understanding that it will not be cited or reproduced without the permission of the author.

This report has been reproduced

directly from the best available copy.

Available to DOE and DOE contractors from the Office of Scientific and Technical Information

P.O. Box 62, Oak Ridge, TN 37831

Prices available from (423) 576-8401 http://apollo.osti.gov/bridge/

Available to the public from the National Technical Information Service

U.S. Department of Commerce 5285 Port Royal Rd., Springfield, VA 22161

http://www.ntis.gov/

OR

Lawrence Livermore National Laboratory Technical Information Department's Digital Library http://www.llnl.gov/tid/Library.html 


\title{
Teller Medal Lecture IFSA2001:
}

\section{Problems and solutions in the design and analysis of early laser driven high energy density and ICF target physics experiments}

\author{
Mordecai D. Rosen \\ Lawrence Livermore National Laboratory \\ University of California \\ Livermore, Ca 94551
}

The high energy density (HED) and inertial confinement fusion (ICF) physics community relies on increasingly sophisticated high power laser driven experiments to advance the field. We review early work in the design and analysis of such experiments, and discuss the problems encountered. By finding solutions to those problems we put the field on firmer ground, allowing the community to develop it to the exciting stage it is in today. Specific examples include: drive and preheat in complex hohlraum geometries with the complicating effects of sample motion; and issues in the successful design of laboratory soft x-ray lasers and in the invention of methods to reduce the required optical laser driver energy by several orders of magnitude.

\section{Introduction}

The field of inertial confinement fusion (ICF) naturally lives in the parameter space of high energy density (HED). Even the initial shock pressure driving a high gain capsule [1] is of order 1 megabar (1 MB) or $10^{12} \mathrm{erg} / \mathrm{cm}^{3}$, followed by successive shocks nearing $100 \mathrm{MB}$ that ultimately accelerates the frozen DT pusher to "thermonuclear speeds" of order $310^{7} \mathrm{~cm} / \mathrm{sec}$. When the DT pusher then stagnates upon implosion, that kinetic energy is converted to thermal energy and fusion ensues, with local pressures exceeding $1 \mathrm{~TB}$ ! In other applications of high power optical lasers such as drivers of exploding foil laboratory soft x-ray lasers [2], dense hot plasmas with internal pressures of some $10 \mathrm{MB}$ serve as the lasing medium.

The field of HED in general, then, relies on increasingly sophisticated high power laser driven experiments in order to advance. In this paper we review early and heretofore unpublished work in the design and analysis of such experiments, and discuss the problems encountered and how solutions were found. In Section 2 we discuss x-ray drive and preheat in complex hohlraum geometries, and the complicating effects of sample motion. Section 3 will review some issues in the successful design of laboratory soft $x$-ray lasers and in the invention of methods to reduce the required optical laser driver energy by several orders of magnitude. Common to these examples was the necessity to look at the problem from a larger, fresh point of view in order to find solutions to the problems. By finding those solutions we put the field of HED studies on firmer ground, allowing the community to develop it to the exciting stage it is in today.

\section{Half-hohIraum HED physics experiments}

\subsection{Experimental Results}

The history of early ICF implosion campaigns is briefly reviewed in Ref [1]. In the mid to late 70's much effort was expended on the 2-beam Argus laser at LLNL to drive glass encased DT spherical capsules indirectly with laser produced x-rays inside a gold cylindrical can or hohlraum. The 2 beams entered on either side of the can through laser entrance holes (LEHs) and impinged on 
on-axis gold cones that were meant to scatter the light in an axisymmetric pattern onto the walls of the can, in the "primary" region, where they would produce x-rays. The capsule sat in the center of the "secondary", between the 2 cones, and radiation flowed to it by passing through the annular region between the on axis scattering cone and the cylinder walls. This was a way to preserve axisymmetry for driving the capsule (under the constraint of having only 2 beams) while not illuminating the capsule directly. The goal of the implosions was to reach the milestone of densities of some $20 \mathrm{gm} / \mathrm{cm}^{3}$ or $100 \mathrm{X}$ liquid density The campaign was called "Cairn" which means milestone.

As the Argus laser was $1.06 \mu \mathrm{m}$ light, we now know in retrospect how challenging a goal "100 X" was, since hot electrons were readily (though somewhat erratically) produced by laser plasma instabilities. These hot electrons caused preheat of the capsule making high-density implosions quite difficult to achieve. However, the inside of a tiny implosion is difficult to diagnose, and doubly so when that implosion is inside a closed can. In order to study the Cairn failure mechanisms in detail, we designed experiments that effectively cut these hohlraums exactly in half, at the mid-plane, and closed off most of the open end with a back plate made of Au wall material. All that was visible then to the outside world, through a $200 \mu \mathrm{m}$ diameter diagnostic hole in the center of the back plate, was the inner surface of the capsule hemisphere. In fact we used a flat slab of glass of equivalent thickness to the capsule pusher instead of a hemisphere. An example of these "half-Cairn" targets is shown in Fig. (1).

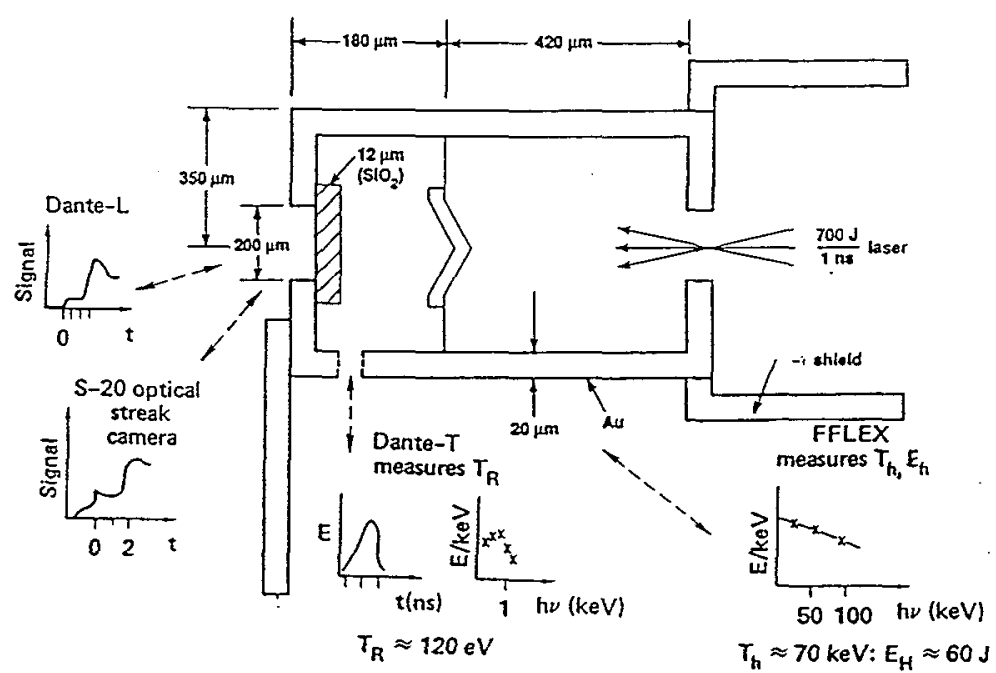

Fig. (1): Experimental set-up and drive side results.

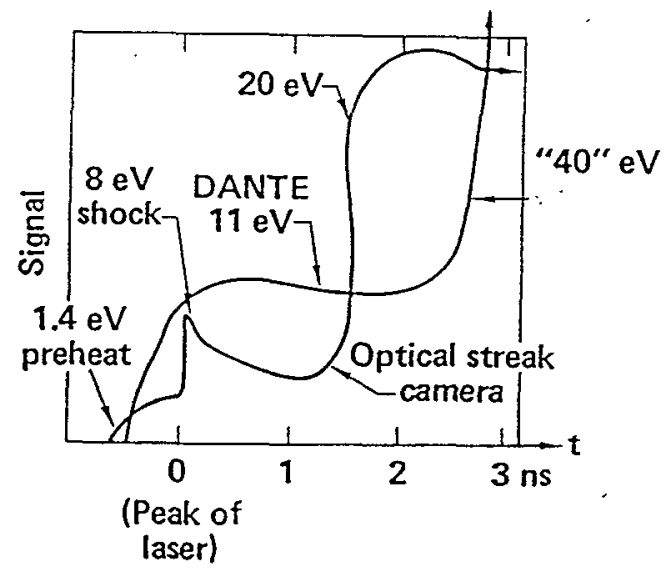

Fig. (2): Cold side data.

The incident laser beam provided about $700 \mathrm{~J}$ in 1 ns to the "half-hohlraum" ("half", of course only in the sense of what the ultimate goal of the study was - understanding implosions in a "full", 2 sided illumination hohlraum. The "half-Cairn" is certainly as much a hohlraum, or x-ray oven, as a full Cairn.). On the "drive" side of the glass slab (namely the side facing the inside of the hohlraum), and local to its surface (namely in the "secondary" part of the half hohlraum, behind the gold scattering cone) we measured the radiation temperature $\left(T_{r}\right)$ to be about $120 \mathrm{eV}$. We used a sub-keV 10 channel, broadband, 250 psec resolution $x$-ray detector named Dante that measures the emission coming from a hole in the side of the hohlraum. (The "primary" region of the half-hohlraum had a $T_{r}$ [measured in a similar fashion] of about $140 \mathrm{eV}$.) Since the Dante was not spatially resolved, the can had large shields on it so that the hole is the only likely $\mathrm{x}$-ray source that Dante can see (the shields block, for example, a plume of x-ray emitting plasma flowing out the LEH). We also measured the production of hot electrons by monitoring the high-energy bremsstrahlung $\mathrm{x}$-rays they produced 
when they stopped in material, with a time and space integrated $x$-ray detector called FFLEX. We inferred that about $60 \mathrm{~J}$ of hot electrons were produced, with a temperature of about $70 \mathrm{keV}$.

On the cold or "burn-thru" side of the glass slab, namely the side facing the outside world, "peeking out" through the $200 \mu \mathrm{m}$ diameter hole in the back plate of the half-hohlraum, we measured the temperature vs. time in 2 ways. We used another Dante spectrometer, and we also used a spatially resolving, absolutely calibrated, 15 ps resolution optical streak camera called the streaked optical pyrometer (or "stroptometer" for short). Fig. (2) shows the results of those 2 measurements. Initially these results raised even more questions than they answered! The early time signals are probably consistent, given the poorer time resolution of the Dante. The stroptometer shows a prompt preheat signal of about $1.4 \mathrm{eV}$ by the laser peak. Then what clearly appears to be a shock sharply breaks out the back to about $8 \mathrm{eV}$. The Dante gives a comparable signal of about $11 \mathrm{eV}$. The mystery seems to be the late time signals. The stroptometer records a "burn-thru" signal of about $20 \mathrm{eV}$ at about $1.5 \mathrm{~ns}$ after the laser peak, whereas the Dante records a $44 \mathrm{eV}$ signal emerging past $2 \mathrm{~ns}$. (The Dante signal is really a flux of $\mathrm{x}$-rays, whose magnitude is interpreted as a temperature by assuming that the signal emerged from the $200 \mu \mathrm{m}$ diameter hole in the back plate of the can through which the cold side of the glass slab is visible). Besides the mystery of the 2 temperatures and 2 break out times, is the overriding mystery that the burn thru signal, if any, was expected to occur much later than either of those 2 times.

Since this was the first time such a physics experiment was attempted with laser driven hohlraums, it was important to get a fundamental understanding of the situation via simple models and rough estimates, besides doing complex 1-D and 2-D simulations with a tool like LASNEX [3]. So let us proceed systematically as we describe the data analysis.

\subsection{Data analysis: Hot electron driven preheat}

As mentioned above, $60 \mathrm{~J}$ of hot electrons with a temperature of about $70 \mathrm{keV}$ were created in the primary region of the hohlraum where the laser interacts with plasma. The question is how much of this impinged onto the glass sample (we'll call it $\mathrm{E}_{\text {inc }}$ for energy incident). To estimate the transport of the hot electrons in the complex half-hohlraum geometry we needed to use both electron number and energy albedoes from both gold and glass of $(0.5,0.7)$ and $(0.13,0.45)$ respectively. Thus, for example, if 100 mono-energetic electrons impinge on glass, 13 are reflected (the rest stick) and have $45 \%$ of their initial energy. We start our crude "transport" calculation with an arbitrary $100 \mathrm{~J}$ worth of hot electrons in the primary. We compute the areas of the primary gold walls, the LEH, and the $A_{P S}$, the annular area around the cone through which the hot electrons can enter the secondary region of the half-Cairn. The electrons are then parceled out to these 3 destinations by the fraction of their areas of that total of the 3, and then the albedoes are applied. Thus after the first iteration, $38.5 \%$ of the original $100 \mathrm{~J}$ worth of hot electrons are reflected from the gold wall back into the primary with 0.7 of their temperature. Another $38.5 \mathrm{~J}$ worth are lost in the gold wall of the primary, as is another $38.5 \mathrm{~J} \times 0.3$ worth (deposited energy by the reflected electrons). Another $3 \mathrm{~J}$ worth are lost out the $\mathrm{LEH}$, and $20 \mathrm{~J}$ worth enter the secondary. The second iteration goes through a similar process, this time both in the primary, and in the secondary. After 3 iterations we find $\mathrm{E}_{\text {inc }}$ equal to $1.2 \mathrm{~J}$ worth of hot electrons impinging on the glass slab at their full initial temperature $(70 \mathrm{keV})$, and $0.8 \mathrm{~J}$ at 50 $\mathrm{keV}$. Had we simply taken the area ratio of the glass sample divided by the total area for the entire half-Cairn, our estimate for $\mathrm{E}_{\text {inc }}$ would have been 3 times higher, and wrong.

This calculation started with an arbitrary $100 \mathrm{~J}$. Since our experiment had $60 \mathrm{~J}$ observed, we reduce our result for $\mathrm{E}_{\mathrm{inc}}$ by a factor of 0.6. Moreover, since the preheat signal is overtaken by the shock signal at the time of the laser peak, only half of the (time integrated result of) $60 \mathrm{~J}$ contributed to the preheat signal at that time, so we multiply $\mathrm{E}_{\text {inc }}$ by another factor of 0.5 .

We can now calculate the expected signal. Taking an ideal equation of state, but supplementing it by adding to the specific heat a factor of 2 to account for ionization energy, and 
another factor of 2 to account for the hydro expansion of the preheated material, our equation for $T_{e}$ reads

$$
\mathrm{T}_{\mathrm{e}}(\mathrm{Z}+1)=3.810^{-5} \varepsilon(\mathrm{J} / \mathrm{gm}) \quad \text { with } \mathrm{Z}=0.8 \mathrm{~T}_{\mathrm{eV}}{ }^{1 / 2} \quad .
$$

Here $\varepsilon$ is the deposited energy due to the hot electrons in J/gm. The range $\lambda$ for the hot electrons is given [4] by:

$$
\lambda=0.3 \mu \mathrm{m}\left(\mathrm{T}_{\mathrm{H}}(\mathrm{keV}) / \mathrm{Z}_{\mathrm{N}}\right)^{2} \mathrm{Z}_{\mathrm{N}}{ }^{1 / 2}
$$

where for our case of glass $Z_{N}=10$, and for $T_{H}=70,50 \mathrm{keV}$, we get $\lambda=50,25 \mu \mathrm{m}$ respectively. These ranges are considerably longer than the $12.4 \mu \mathrm{m}$ thickness of the glass. Therefore, the $\varepsilon$ of Eq. (1) is simply $E_{\text {inc }}$ divided by the respective $\lambda \mathrm{s}$, and divided by the density $\rho$ of the glass $\left(2.5 \mathrm{~g} / \mathrm{cm}^{3}\right)$ and by the area of the glass sample $\left(710^{-4} \mathrm{~cm}^{2}\right)$. Then Eq. (1) gives $T_{e}$ of $1.7 \mathrm{eV}$, in close agreement with the observed $1.4 \mathrm{eV}$.

\subsection{Data analysis: $\mathrm{X}$-ray driven shock waves}

As mentioned in Sec. 2.1, the measure drive was $T_{P}=140 \mathrm{eV}$ in the primary region of the hohlraum and, more relevantly, $\mathrm{T}_{\mathrm{s}}=120 \mathrm{eV}$ in the secondary region which drives the glass slab. Because of length limitations on this manuscript we will not reproduce here a detailed worked example, for precisely our situation, that has already been published in reference [5]. There we worked out a model based on simple energy balance in the primary and secondary region ( $\mathrm{x}$-ray sources balanced by energy losses of a diffusive Marshak wave into the walls, as well as loss out the LEH and into the glass sample). Our quantitative estimates for $T_{p}$ and $T_{S}$ were found there to be in close agreement with the observations.

Working through the radiation ablation hydrodynamics in glass can lead to a prediction for the pressure generated by such a process: $P=8 \mathrm{~T}_{\mathrm{heV}}{ }^{3} \mathrm{t}_{\mathrm{ns}}{ }^{-0.4} \mathrm{MB}$. Thus for our parameters $\mathrm{T}=\mathrm{T}_{\mathrm{S}}=$ $1.2 \mathrm{heV}$, and $\mathrm{t}_{\mathrm{ns}}=1$, we expect a $14 \mathrm{MB}$ shock to propagate through the glass slab. This strong shock should quadruple the pre-shock density, and thus we estimate the post shock temperature via

$$
\mathrm{T}_{\mathrm{e}}(\mathrm{Z}+1)=\left(\mathrm{A} / 4 \rho_{0}\right) \mathrm{P}_{\mathrm{MB}} \quad \text { again with } \mathrm{Z}=0.8 \mathrm{~T}_{\mathrm{eV}}{ }^{1 / 2}
$$

where $A$ is the atomic weight of glass of 20 . Thus with a $14 \mathrm{MB}$ shock, we expect a $9.5 \mathrm{eV}$ shock temperature, which agrees well with the measured 8-11 eV. Estimating the shock velocity by the strong shock relations $\mathrm{v}_{\mathrm{s}}{ }^{2}=4 \mathrm{P} / 3 \rho_{0}$, gives $\mathrm{v}_{\mathrm{s}}=310^{6} \mathrm{~cm} / \mathrm{s}$. Thus the shock will traverse the 12.4 $\mu \mathrm{m}$ glass in about $400 \mathrm{psec}$, breaking out the back well within the $1 \mathrm{~ns}$ laser pulse width, as is indeed observed.

One dimensional LASNEX simulations of this experiment, corrected for the hot electron transport factors discussed above, indeed confirm all of our simple estimates, and reproduces the early time cold side of the glass preheat and shock temperatures. However those results also show that the cold side is not predicted to "burn-thru" and should stay at the $10 \mathrm{eV}$ level. What then, accounts for the mysterious late time signals of Fig. (2)? Stepping back, and taking a larger, fresh view of the situation brought us answers.

\subsection{Data analysis: X-ray driven sample motion: "Cork popping"}

When the shock wave breaks out of the back of the glass, a rarefaction wave propagates back to the radiation ablation front. A pressure gradient is then set up which accelerates the "payload" the unablated portion of the glass slab-as a whole. The central $200 \mu \mathrm{m}$ portion of the glass is then "cookie cut" out of the hole in the back of the hohlraum, and moves out of the hohlraum. This "popped cork" then releases hot ablated gas that was previously "bottled up" within the hohlraum and makes it visible to the 2 detectors. Thus, indeed the glass slab does not burn through, but rather allows the "cold side" detectors to see hot internal plasma as the popped cork's cold side moves out of the way of their line of sight. Since the detectors view the cold side at 2 different angles, they see a hot late-time signal at different times, corresponding to the cold popped cork moving out of their respective lines of sight. A schematic that illustrates this concept is shown in Fig. (3). 
Let us estimate the effect. The amount of glass ablated, again calculated by working through the radiation ablation hydrodynamics in glass is given by $\delta \mathrm{x}=\mathrm{m} / \rho_{0}=910^{-4} \mathrm{~T}_{\mathrm{hev}}{ }^{2.25} \mathrm{t}_{\mathrm{ns}}{ }^{0.6} / \rho_{0}(\mathrm{~cm})$. Thus for our parameters $(\mathrm{T}=1.2, \mathrm{t}=1)$ we get $\delta \mathrm{x}=5.4 \mu \mathrm{m}$ ablated. The payload acceleration will then be given by $a=F / m=P / \rho_{0}(12.4 \mu \mathrm{m}-\delta x)$ where for our case of $14 \mathrm{MB}$ pressure, $a=810^{15} \mathrm{~cm}^{2} / \mathrm{s}$. We now can calculate the distance the payload will move as a function of time:

$$
\mathrm{d}=1 / 2 \mathrm{at}_{\mathrm{a}}{ }^{2}+\mathrm{at}_{\mathrm{a}} \mathrm{t}=10 \mu \mathrm{m}+40(\mu \mathrm{m} / \mathrm{ns}) \mathrm{t}_{\mathrm{ns}}
$$

where we take the acceleration time $t_{\mathrm{a}}$ to be $1 / 2 \mathrm{~ns}$ because the shock does not break out the back until the peak of the laser when half the $1 \mathrm{~ns}$ pulse is over. To estimate the late time signals of the 2 detectors we note that the stroptometer is at $55^{\circ}$ from the target normal, so the $100 \mu \mathrm{m}$ radius, cold payload slug must travel $70 \mu \mathrm{m}$ before the stroptometer can see past it into the hot hohlraum. The Dante is at $45^{\circ}$ so the cork must pop out $100 \mu \mathrm{m}$ from the can before the Dante can see past its cold side into the hot hohlraum. Setting $d=70,100 \mu \mathrm{m}$ in Eq. (4) and solving for $t$ leads to predictions of late signals of $1.5,2.25 \mathrm{~ns}$ for the 2 detectors respectively. These times are quite close to those observed as per Fig. (2). Our 1-D LASNEX simulations confirm our estimates of this payload motion as well.

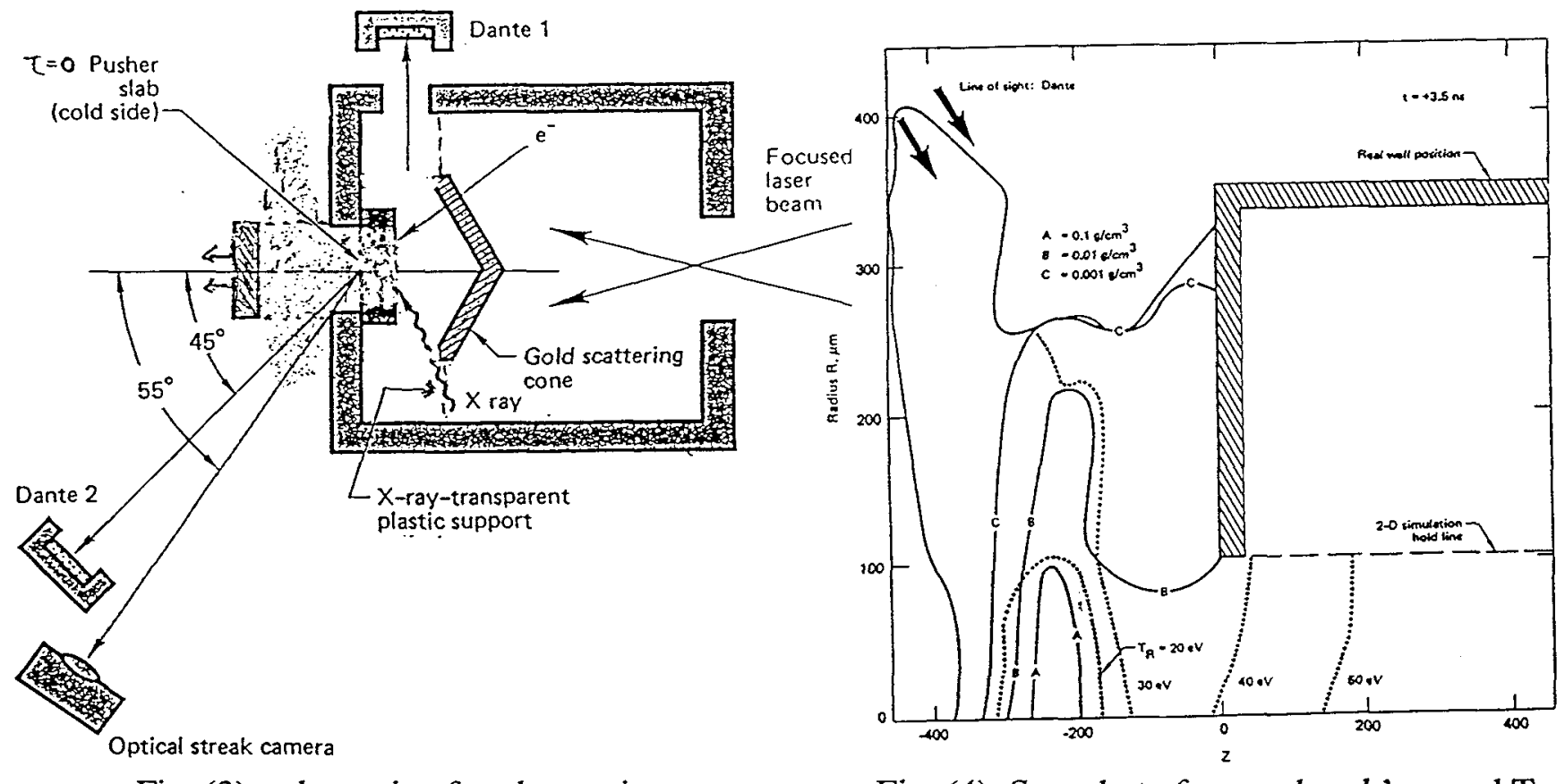

Fig. (3): schematic of cork popping.

Fig. (4): Snapshot of popped cork's $p$ and T.

We are left with one remaining mystery. Why do the 2 detectors differ in the temperature they see at late times? Here 2-D LASNEX simulations help us with the answer. In Fig (4) we show a snapshot in time of the densities and temperatures of the popped cork and its surrounding hot plasma liberated from the hohlraum. The Dante line of sight passes through material of density $\rho=510^{-3}$ $\mathrm{g} / \mathrm{cm}^{3}$ and temperature of $25-30 \mathrm{eV}$. The $100 \mathrm{eV}$ photons detected by Dante have an opacity $\mathrm{K}$ of about $310^{4} \mathrm{~cm}^{2} / \mathrm{g}$ in such a plasma which means their mean free path, $1 / \mathrm{kp}$, is $66 \mu \mathrm{m}$. Thus this hot gas liberated by cork popping is optically thick. Dante does not see all the way into the small hot exit hole, but rather (recall it is not spatially resolved) sees a much larger area radiating at $25-30 \mathrm{eV}$. Indeed as Fig (5) shows, a complete post processing of the 2-D LASNEX simulation, mimicking the Dante detector and all of its channels, shows that the emission of $100 \mathrm{eV}$ photons, (dark regions in the figure) come from a large area "halo" around the cold (low emission, bright region in the figure) image of the $200 \mu \mathrm{m}$ diameter glass plug's cold side. Moreover, in Fig. (6) we show the simulated Dante spectrum "collected" from that snapshot in time of the hot glass plasma of Figs. (4) and (5), 
which very closely reproduces the 5 broad Dante channels of data. Note that the spectrum has a color temperature of about $25 \mathrm{eV}$. We can integrate the energy of that spectrum, and get a total flux. If we assume misleadingly (as Dante did originally when quoting its temperature of $44 \mathrm{eV}$ ) that the flux is all coming from the small $200 \mu \mathrm{m}$ diameter hole in the back plate, then LASNEX too would characterize that flux with a "brightness" temperature of $44 \mathrm{eV}$. The stroptometer is spatially resolved, so it was not "fooled", and reported the temperature of the optically thick liberated gas correctly, as $20 \mathrm{eV}$.

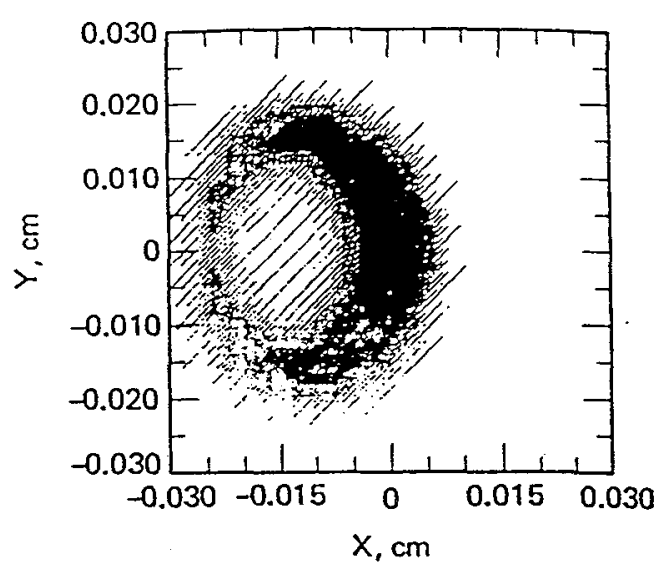

Fig. (5): Simulated cold-side image.

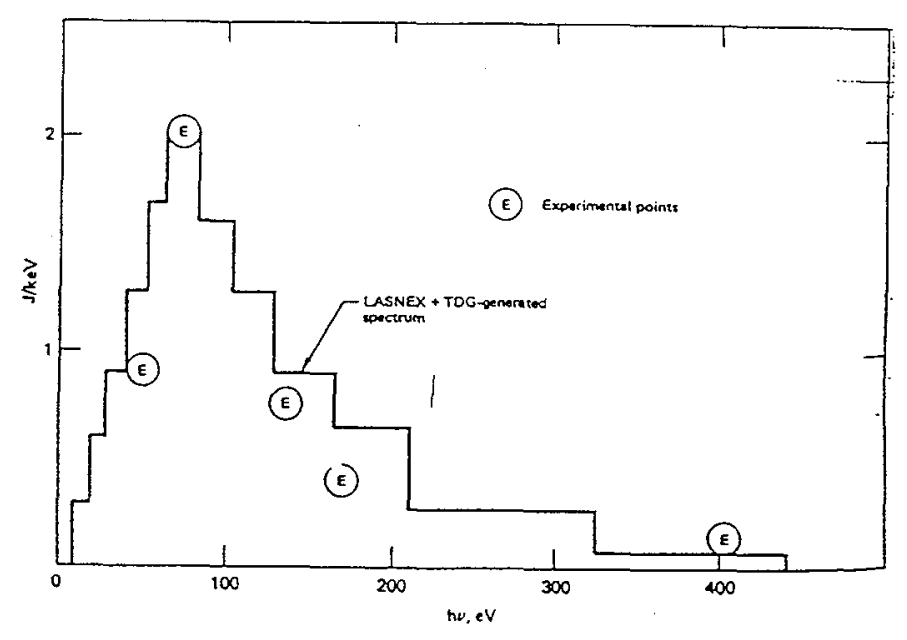

Fig. (6): Simulated cold-side spectrum vs. data.

\subsection{Implications of the work}

The shift from bewilderment to total understanding of the cold-side burn-thru signals in this, the first half-hohlraum physics experiment, gave a big boost of confidence to workers in the field, and spurred them on to make further rapid progress. Preheat detection was soon augmented by spatially resolving $\mathrm{K} \alpha$ emission diagnostics. These later helped identify $\mathrm{B}$ fields in hohlraum plasmas and have played a large role in fast ignitor research. Shock wave detection progressed from single slab to the more accurate stepped slab to measure shock velocities. That evolved further to wedge shaped slabs [6] to monitor shock velocity continuously, thus allowing for accurate pulse shaping data. Shocks were also produced by one $x$-ray driven plate colliding into another [7], acting as a power amplifier by collecting energy over a long time, storing it as kinetic energy, and delivering it rapidly back to thermal pressure during the collision. This led to very high shock pressures being achieved [8]. Further experimental refinements led to the ability to do detailed equation of state measurements [9], and material strength measurements at high pressure [10]. The cork popping was a "poor man's" way of measuring pusher speed. Soon, x-ray backlighting took over as a much clearer way to do so [11]. That soon led to quantitative measurements of hydro instabilities [12], and eventually to the mocking up of such exotic phenomenon as astrophysical jets from galactic centers[13]! While in these initial half-Cairn experiments we never really did "burnthru", we learned enough to redesign experiments that really did have bona-fide radiation burn-thru signals, which taught us much about material opacities [5]. We used the same technique to improve hohlraum energetics by testing "cocktail" materials [14] that were combinations of elements that scattered $x$-rays more effectively back into the hohlraum, and burned through later in time. Eventually, exquisitely detailed frequency dependant opacities were also measured in half hohlraums as well [15]. In addition, the "ultimate" in burn-thru experiments (due to the minor role 
hydrodynamic motion plays in it) supersonic, diffusive radiative heat flow through low density high $\mathrm{Z}$ foams, driven by half hohlraums have also been achieved[16].

Thus this body of work has contributed to understanding ICF target physics and performance, and to HED science, with astrophysics as a particular application. Moreover, this body of work, already maturing by the early 90 's, allowed the US national weapons lab directors to recommend that the US government attempt the experiment known as science based stockpile stewardship[17], whose most salient components are the cessation of nuclear testing, the creation of even larger HED facilities such as the National Ignition Facility, and the establishment of modern supercomputing capabilities.

\section{Laboratory X-ray lasers}

\subsection{Solving the initial problems}

By the mid 80's there had been many years of attempts at producing a soft $x$-ray laser in the laboratory, without success. Our first challenge was to guess what were the true reasons for those failures. The atomic physics predictions for population inversions were based on complex calculations. Could they be wrong? We took a different tack. We assumed the atomic physics predictions were reasonably accurate, and concentrated on the propagation of the $\mathrm{x}$-ray laser pulse. Refraction in steep density gradients could rapidly steer the beam out of the gain medium, thus lowering the effective gain to the realm of the undetectable. We adapted a target from our ICF experience- exploding foils [2] that produced relatively uniform scale lengths, of order $100 \mu \mathrm{m}$, which would be sufficient to greatly lessen beam steering, and allow the effective gain to be close to its theoretical values. This turned out to be a correct guess, and a successful strategy. Analogous to the ICF work described in Sec. 2 above, we supplemented our successful full blown XRL experiments with physics experiments of the exploding foils themselves, diagnosing them in space and time, and successfully predicting that behavior, both with simple analytic models [18], and with complex LASNEX simulations.

After our initial successes on the Novette green light laser ( 2 beams of the Nova laser) in 1984, producing about $100 \mathrm{~W}$ of $200 \AA$ radiation from 3p-3s Ne-like Se [19], we made rapid progress. By the end of the 80's we had upped that output to $1 \mathrm{MW}$ [20], and demonstrated lasing at sub $100 \AA$ with $\mathrm{Ne}-$ like $\mathrm{Ag}$ [21]. We used an analog to that scheme, the $4 \mathrm{~d}-4 \mathrm{p}$ Ni-like scheme [22] in Ta to demonstrate gain [23] at the 45 $\AA$ quite relevant for holography of biological samples in water. Our technologists developed $x$-ray optics, so a triple pass cavity was demonstrated [24], and initial $x$-ray imaging applications were accomplished as well $[25,26]$. However we knew that the field would not really flourish into one filled with practical applications if the driver remained the huge 2 beams of Nova [27]. Thus, once again, we needed to step back, take a larger look at what we were doing, and find a fresh way to accomplish our goals.

\subsection{Solving the next generation of problems: towards tabletop lasing}

The key to our initial success was the uniform lasing medium, so we asked ourselves if we could pre-form that medium at minimal energy cost. Once formed, could we "flash heat" it to lasing conditions, using the short pulse (low energy but high power) laser technology that was becoming small, cheap, and commonplace in the late 80 's. By way of comparison, the conventional way to achieve $40 \AA$ saturated (gain-length product of about 15 ) lasing with Ni-like W, would require a 1.5 $\mathrm{cm}$ long foil, with a gain coefficient of $10 \mathrm{~cm}^{-1}$. That foil, in our conventional way, would be illuminated for $1 \mathrm{~ns}$, at $610^{14} \mathrm{~W} / \mathrm{cm}^{2}$ with a line focussed $100 \mu \mathrm{m}$ by $1.5 \mathrm{~cm}$ spot. This translates to 9 $\mathrm{kJ}-\mathrm{a}$ hugely expensive proposition. If instead we simply formed the plasma with a $1 \mathrm{~ns}, 1.5 \mathrm{~cm}$ by $50 \mu \mathrm{m}$ line focus spot, at the very low irradiance of $210^{12} \mathrm{~W} / \mathrm{cm}^{2}$, it could produce, over time the correct density profile that would allow propagation without refraction. That translated into a mere $15 \mathrm{~J}$ of energy. Then, at that proper time when the density gradient was "right", we'd bring in a short 
20 ps pulse, tightly focussed to $1.5 \mathrm{~cm}$ by $30 \mu \mathrm{m}$, at the proper high irradiance of $610^{14} \mathrm{~W} / \mathrm{cm}^{2}$ to create the Ni-like state and the population inversion. That would require $54 \mathrm{~J}$. All told, more than a 100 fold savings in required driver energy, and a true tabletop system. Our detailed calculations [28] of such a scheme are shown in Fig (6), and confirmed our notions of its feasibility. Note that the gain coefficient in the center of the foil is predicted to be nearly $10 \mathrm{~cm}^{-1}$, as per our assumptions above. Of course it takes the XRL beam 50 ps to propagate down the full $1.5 \mathrm{~cm}$ length of the plasma medium, whereas the gain only lasts about 20 ps. Thus the 20 ps pump pulse will have to be "phase driven" down the $1.5 \mathrm{~cm}$ length of the plasma medium. There are well known techniques for doing this [29]. We also realized [28, 30] that capillary discharges could also be a good tabletop approach. The mid to late 90's featured several groups [31,32] succeeding in making tabletop XRLs using these schemes, thus making it far more likely that practical applications will one day be commonplace.
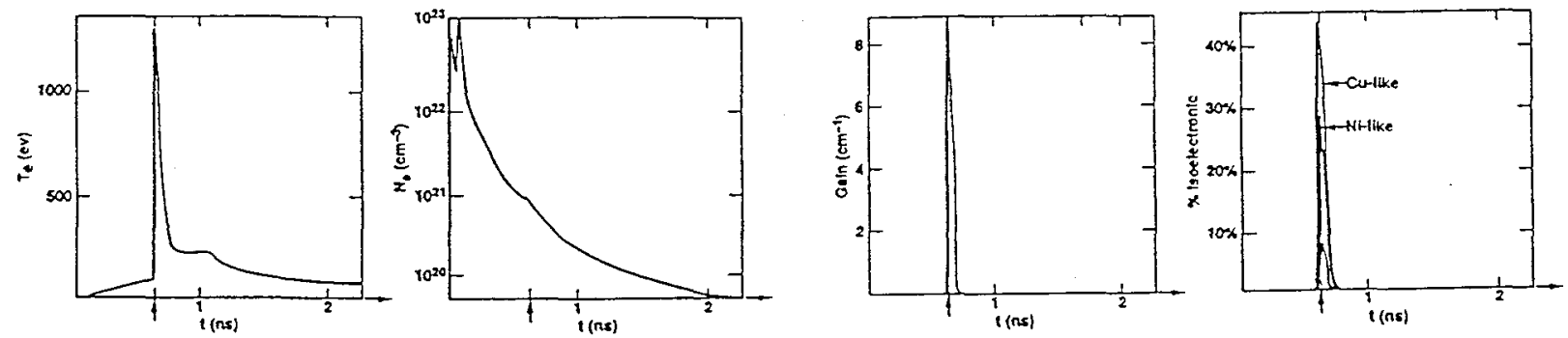

Fig. (6): At foil center, $\mathrm{T}_{\mathrm{e}}, \mathrm{n}_{\mathrm{e}}$, gain, \& \% ion-state vs. time. Arrow indicates onset of short pulse

\subsection{Summary and Conclusions}

The ICF/HED half-hohlraum work described in Sec. 2, and the XRL work of Sec. 3 share several features in common. Initial failures of the integrated experiment, be it high-density implosion, or actual XRL, led to designs and tests of physics experiments. Both simple models and complex simulations were important tools in those designs and in the data analysis. Solving the initial problems with those physics experiment required looking at the problem from a larger, fresh point of view. The ensuing very successful development of these fields has been most gratifying, and is a tribute to our many coworkers in these fields, which we now proceed to acknowledge.

\subsection{Acknowledgements}

For the half Cairn work we are grateful to: W. Mead for the initial designs, G. Tirsell and D. Banner for campaign leadership, D. Phillion, B. Price, B. Kauffman, H. Kornblum, L. Koppel, and C. Wang for diagnostic development and fielding, M. Boyle, L. Coleman and E. Storm for experimental guidance, J. Lindl and J. Nuckolls for theoretical support, B. Thomas, S. Davidson, A. Szoke, C. Alonso, and R. Ward for design/spin-off ideas, and D. Munro, B. Hammel, B. Remington, R. Collins, G. Dimonte, T. Peyser, R. Klein and P. Springer for leadership in subsequent developments.

For the XRL work we are grateful to: D. Matthews for experimental leadership, P. Hagelstein, for atomic theory / lasing scheme leadership, M. Campbell, R. London, S. Maxon and D. Eder for design support, B. MacGowan, L. Da Silva, M. Eckart, J. Trebes, N. Ceglio, R. Johnson, and G. Charatis for experimental support, A. Hazi, B. Whitten, J. Scofield, D. Lee, B. Goldstein and T. Weaver for atomic physics support, B. Hatcher, G. Rambach and G. Stone for target fabrication, and J. Rocca, J. Nilsen, S. Libby, A. Wan and J. Dunn for subsequent developments. 
For computational help, and in particular, for the LASNEX simulation code support, we are indebted to: G. Zimmerman, D. Bailey, J. Harte, D. Kershaw, H. Shay, J. Larsen, M. Prasad, A. Shestakov, and E. Alley.

For general guidance and support we thank J. Nuckolls, J. Lindl, B. Tarter, W. Lokke, J. Emmett, G. Miller, D. Fortner, M. Campbell, J. Kilkenny, R. Woodruff, J. Wadsworth, M. Anastasio, C. Verdon, R. Ward, B. Goodwin, M. May, R. Batzel and E. Teller.

This work was performed at LLNL under the auspices of the U.S. DoE under contract No. W-7405-ENG-48.

\section{References}

1. J. D. Lindl, Phys. Plasmas 2, 3933 (1995).

2. M. D. Rosen, et. al Phys. Rev. Lett. 54, 106 (1985).

3. G. B. Zimmerman and W. L. Kruer, Comm. in Plasma Physics 2, 85 (1975).

4. M. D. Rosen, et. al. Phys. Rev A 36, 247 (1987).

5. M. D. Rosen, Phys. Plasmas 7, 1999 (2000).

6. R. L. Kauffman, et. al Phys. Rev. Lett. 73, 2320 (1994).

7. M. D. Rosen, et al., in "Shock Waves in Condensed Matter - 1983" J. R. Asay, G. K. Straub, and R. A. Graham, Editors, North-Holland Publishing Co. (Amsterdam), 1984, pg. 323.

8. R. C. Cauble et al., Phys. Rev. Lett. 70, 2102 (1993).

9. G.W. Collins et al., Science 281, 1178 (1998).

10. D. H. Kalantar, et al., Phys. Plasmas 3, 1803 (1996).

11. R. H. Price, et al., in "Shock Waves in Condensed Matter - 1981" W. J. Nellis,L. Seaman, and R. A. Graham, eds., American Institute of Physics 78, 155 (1982).

12. J. D. Kilkenny, Phys. Fluids B 2, 1400 (1990).

13. B. A. Remington, et al., Science 284, 1488 (1999).

14. T. J. Orzechowski, et al., Phys. Rev. Lett. 77, 3545 (1996).

15. P. T. Springer, et al., Phys. Rev. Lett. 69, 3735 (1992).

16. C. A. Back, et al., Phys. Rev. Lett. 84, 274 (2000).

17. R. Jeanloz, Physics Today 53, 44 (2000).

18. R. A. London and M. D. Rosen, Phys. Fluids 29, 3813 (1986).

19. D. L. Matthews, et al., Phys. Rev. Lett. 54, 110 (1985).

20. B. J. MacGowan, et al., Phys. Fluids B 4, 2326 (1992).

21. D. J. Fields, et al., Phys. Rev. A 46, 1606 (1992).

22. M. S. Maxon, et al., Phys. Rev. Lett. 63, 236 (1989).

23. B. J. MacGowan, et al., Phys. Rev. Lett. 65, 420 (1990).

24. N. M. Ceglio, et al., Opt. Lett. 13, 108 (1988).

25. L. B. Da Silva, et al., Science 258, 269 (1992).

26. D. M. Ress, et al., Science 265, 514 (1994).

27. D. C. Eder, Phys. Plasmas 1, 1744 (1994).

28. M. D. Rosen, OSA Proceedings on Short Wavelength Coherent Radiation: Generation and Applications, P. Buchsbaum and N. Ceglio eds. (OSA, Wash. DC 1991) Vol. 11, pg. 96.

29. Z. Bor, et al., Appl. Phys. B. 32, 101 (1983).

30. M. D. Rosen and D. L. Matthews, U.S. Patent \# 5016250 (5/14/1991).

31. J. J. Rocca, et al., Phys. Rev. Lett. 77, 1476 (1996).

32. J. Dunn, Phys. et al., Phys. Rev. Lett. 84, 4834 (2000). 